\title{
FORME TRACE ET RAMIFICATION SAUVAGE
}

\author{
CHRISTINE BACHOC et BOAS EREZ
}

[Received 8 February 1989]

\begin{abstract}
Let $A(K / \mathbb{Q})$ denote the fractional ideal of a cyclic $p$-extension $K / \mathbb{Q}$ whose square is the inverse different of the extension. Equipped with the trace form, $A(K / \mathbb{Q})$ becomes a $\mathbb{Z} G a l(K / \mathbb{Q})$-hermitian module $\left(A(K / \mathbb{Q}), \operatorname{Tr}_{K / Q}\right)$ with discriminant 1 . By using results on the Galois module structure of $A(K / \mathbb{Q})$ and a classification of forms in cyclotomic fields, we show that if $p$ is totally-and hence wildly-ramified in $K / \mathbb{Q}$, then the equivariant isometry class of $\left(A(K / \mathbb{Q}), \operatorname{Tr}_{K / \mathbb{Q}}\right)$ depends only on the degree of the extension.
\end{abstract}

\section{Introduction}

Soit $K / \mathbb{Q}$ une extension galoisienne de groupe de Galois $G$ et de degré fini impair. Dans cet article nous poursuivons l'étude du $\mathbb{Z} G$-réseau $\left(A_{K}, \operatorname{Tr}_{K / \mathbb{Q}}\right)$ obtenu en restreignant la forme trace

$$
\operatorname{Tr}_{K / \mathbb{Q}}: K \times K \rightarrow \mathbb{Q}, \quad \operatorname{Tr}_{K / \mathbb{Q}}(x, y)=\operatorname{trace}_{K / \mathbb{Q}}(x y),
$$

de l'extension $K / \mathbb{Q}$ à l'unique idéal $A_{K}$ de $K$ dont le carré est la codifférente $D(K / \mathbb{Q})^{-1}$. Rappelons que d'une part l'idéal $A_{K}$ est le seul idéal rendant la forme trace unimodulaire (voir $\$ 1.4$ ), et que d'autre part la codifférente étant stable par l'action de $G, A_{K}$ est un $\mathbb{Z} G$-module.

Soit $\left(\mathbb{Z} G, T_{1}\right)$ le $\mathbb{Z} G$-réseau où $T_{1}$ est la forme sur l'algèbre de groupe $\mathbb{Q} G$ pour laquelle les éléments de $G$ forment une base orthonormale. Dans [6-8] on compare $\left(A_{K}, \operatorname{Tr}_{K / \mathbb{Q}}\right)$ et $\left(\mathbb{Z} G, T_{1}\right)$, et en particulier on se demande quand ils sont isométriques.

Par définition une condition nécessaire pour l'existence d'une isométrie équivariante $\left(A_{K}, \operatorname{Tr}_{K / \mathbb{Q}}\right) \sim\left(\mathbb{Z} G, T_{1}\right)$ est que le module galoisien $A_{K}$ soit isomorphe à $\mathbb{Z} G$. Il est montré dans [6-8] que si $G$ est abélien, alors $A_{K}$ est $\mathbb{Z} G$-libre si et seulement si l'extension $K / \mathbb{Q}$ est peu ramifiée: une extension abelienne $K / \mathbb{Q}$ est dite peu ramifiée si les premiers $p$ de $\mathbb{Q}$ qui se ramifient sauvagement dans $K / \mathbb{Q}$ ont un indice de ramification égal à $p: e(p)=p$. Il s'avère que dans le cas abélien l'isomorphisme de $\mathbb{Z} G$-modules entre $A_{K}$ et $\mathbb{Z} G$ (sans formes) entraîne l'existence d'une isométrie équivariante. En résumé on a donc le

THÉORÈME 0.1 [6-8]. Soit $K / \mathbb{Q}$ une extension abélienne. Il existe une isométrie équivariante entre $\left(A_{K}, \operatorname{Tr}_{K / \mathbb{Q}}\right)$ et $\left(\mathbb{Z} G, T_{1}\right)$ si et seulement si $K / \mathbb{Q}$ est peu ramifiée.

Notre but est de montrer un analogue du Théorème 0.1 dans une situation où $K / \mathbb{Q}$ est très sauvagement ramifiée. Plus précisément on va considérer des extensions galoisiennes $K / \mathbb{Q}$ telles que:

$(0.2)$ le groupe de Galois $G=\mathrm{Gal}(K / \mathbb{Q})$ est un groupe cyclique d'ordre $p^{n}$, où $p \neq 2$ est un nombre premier, et $n$ est un entier naturel, et de plus $p$ se ramifie totalement dans $K / \mathbb{Q}$.

Ce travail a été effectué lorsque le deuxième auteur bénéficiait d'une bourse du F.N.R.S. Suisse et de l'hospitalité de l'U.E.R. de Mathématiques de l'Université de Bordeaux 1.

A.M.S. (1980) subject classification: 12A57, $10 \mathrm{C02}$.

Proc. London Math. Soc. (3) 61 (1990) 209-226. 
Remarquons qu'aucune hypothèse n'est faite sur la ramification des nombres premiers autres que $p$.

Nous allons montrer le

THÉORÈmE 0.3 . Soient $K / \mathbb{Q}$ et $K^{\prime} / \mathbb{Q}$ deux extensions galoisiennes vérifiant $(0.2)$. Alors pour toute identification des groupes de Galois $\mathrm{Gal}(K / \mathbb{Q})$ et $\mathrm{Gal}\left(K^{\prime} / \mathbb{Q}\right)$ on a une isométrie équivariante entre $\left(A_{K}, \operatorname{Tr}_{K / Q}\right)$ et $\left(A_{K^{\prime}}, \operatorname{Tr}_{K^{\prime} / Q}\right)$.

La démonstration de ce théorème va suivre d'une description explicite d'un représentant 'abstrait' de la classe d'isométrie équivariante de $\left(A_{K}, \operatorname{Tr}_{K / Q}\right)$. Ainsi par exemple si l'ordre de $G$ est $p$, l'extension est peu ramifiée et nous allons retrouver le fait que $A_{K}$ possède une base orthonormale, i.e., $A_{K}$ est isométrique à la forme standard $\langle 1, \ldots, 1\rangle$. Plus généralement si $n$ est pair (respectivement impair) alors $A_{K} \sim\langle 1\rangle \oplus \mathscr{B}_{n}$ (respectivement $A_{K} \sim\langle 1, \ldots, 1\rangle \oplus \mathscr{B}_{n}$ ) où $\mathscr{B}_{n}$ est une forme unimoduiaire, définie positive, paire, de rang $p^{n}-1$ (respectivement $p^{n}-p$ ). Les vecteurs de longueur 2 dans $\mathscr{B}_{n}$ engendrent un système de racines $\operatorname{Rac}\left(\mathscr{B}_{n}\right)$ qui est de rang maximal; nous décrivons $\mathscr{B}_{n}$ dans la Proposition 6.6 et nous calculons $\operatorname{Rac}\left(\mathscr{B}_{n}\right)$ dans la Proposition 6.9.

Esquissons la preuve du Théorème 0.3. Soit $\mathcal{M}$ l'ordre maximal de $\mathbb{Q} G$. On commence par remarquer que les hypothèses $(0.2)$ permettent de se ramener au calcul de la classe d'isométrie équivariante du plus petit sous-module de $K$ contenant $A_{K}$ qui soit stable par $\mu$ (voir $\S 3$ ). Ce module sera noté $M A_{K}$. Il est essentiel pour cette étape de réduction que l'extension soit très sauvagement ramifiée et qu'un seul premier divise l'indice $\left[M A_{K}: A_{K}\right]$. Il s'agit ensuite de montrer que la classe d'isométrie équivariante de $\left(M A_{K}, \operatorname{Tr}_{K / Q}\right)$ ne dépend que des conditions (0.2). Nous utilisons le fait que $M A_{K}$ est libre sur $\mathcal{M}$ (voir [8]), et nous nous ramenons à l'étude des formes sur $\mathcal{M}$.

On sait, que pour $G$ cyclique d'ordre $p^{n}, M \cong \bigoplus_{i=0}^{n} \mathbb{Z}\left[p^{i}\right]$, où $\mathbb{Z}\left[p^{i}\right]$ est l'anneau des entiers du corps cyclotomique $\mathbb{Q}\left(p^{i}\right)$ des racines $p^{i}$-èmes de l'unité. Nous sommes donc amenés à étudier des formes équivariantes dans les corps cyclotomiques et au $\S 4$ nous allons classer certaines formes $\mathbb{Z}\left[p^{i}\right]$-équivariantes sur un idéal de $\mathbb{Q}\left(p^{i}\right)$ par un groupe $\mathscr{E}$ (voir la Proposition 4.2 et le Corollaire 4.6). A ce stade nous aurons besoin de la description en termes de sommes de Gauss des résolvantes d'un générateur libre de $M A_{K}$ sur $M$. Cette description nous permettra de repérer la forme qui nous intéresse dans le groupe $\mathscr{E}$.

En résumé notre travail aura donc consisté-encore une fois-à déduire la structure quadratique d'une connaissance précise de la structure galoisienne. Nous disons à la Remarque 5.6 comment on retrouve de la même manière les résultats de Conner et Perlis sur l'anneau des entiers (voir [4, Chapter IV]). Remarquons aussi que les calculs du $\S 2$ permettent de déterminer l'ordre associé à $A_{K}$.

Le paragraphe 1 contient les définitions et les notations nécessaires. Le dernier paragraphe contient des exemples.

Après la rédaction de cet article nous nous sommes rendus compte que beaucoup d'idées que nous utilisons sur le lien entre forme trace et résolvantes se trouvent déjà dans l'oeuvre de $\mathrm{A}$. Fröhlich et sont à la base de la théorie de son 'Pfaffien'. Nous invitons le lecteur à considérer notre travail comme une introduction à [11] et nos résultats comme un type de conséquences qu'il serait souhaitable de pouvoir en tirer. 
Remerciements. Les auteurs remercient Jacques Martinet et Jacques Queyrut pour de stimulants entretiens ainsi que Eva Bayer pour les conseils permettant d'étendre les résultats de [13] à la situation plus générale considérée au $\$ 4$.

\section{Définitions et notations}

\subsection{Réseaux}

Soit $G$ un groupe fini, $F$ un corps de nombres et $\mathbb{Z}_{F}$ l'anneau des entiers de $F$. On s'interesse aux couples $(V, b)$ où $V$ est un $F G$-module de dimension finie sur $F$ muni d'une forme

$$
b: V \times V \rightarrow F
$$

bilinéaire, symétrique et $G$-équivariante. C'est-à-dire que pour tout $x$ et $y$ dans $V$ et pour tout $g$ dans $G$,

$$
b(g(x), g(y))=b(x, y) .
$$

Toutes les formes considérées dans la suite seront non-dégénérées, c'est-à-dire d'adjoint injectif.

Un $\mathbb{Z}_{F} G$-réseau $(R, b)$ dans $(V, b)$ est un $\mathbb{Z}_{F} G$-module $R$ contenu dans $V$ et tel que $F R=V$. Soit $R=(R, b)$ un $\mathbb{Z}_{F} G$-réseau dans $(V, b)$ :

-le réseau dual de $R$ est le $\mathbb{Z}_{F} G$-réseau

$$
R^{*}=\left\{x \in F R \mid b(x, R) \subseteq \mathbb{Z}_{F}\right\}
$$

On sait que $\left(R^{*}\right)^{*}=R$.

$-R$ est dit entier si $R \subseteq R^{*}$, ou de façon équivalente, si pour tout $x$ et $y$ dans $R$, $b(x, y)$ appartient à $\mathbb{Z}_{F}$;

-si $R$ est entier on définit le discriminant de $R$ comme étant l'indice

$$
\operatorname{disc}(R):=\left[R^{*}: R\right],
$$

c'est un entier positif si $F=\mathbb{Q}$.

$-R$ est unimodulaire si $R=R^{*}$.

Soit maintenant $F=\mathbb{Q}$ :

-le réseau entier $R$ est pair si pour tout $x$ dans $R$ l'entier $b(x, x)$ est pair;

$-R$ est indécomposable s'il l'est en tant que $\mathbb{Z} G$-module.

La forme $b$ est définie positive s'il existe une isométrie définie sur le corps des réels. $\mathbb{R}$ entre $b$ et la forme standard $\langle 1, \ldots, 1\rangle$.

Deux $\mathbb{Z} G$-réseaux $R$ dans $(V, b)$ et $R^{\prime}$ dans $\left(V^{\prime}, b^{\prime}\right)$ sont $\mathbb{Z} G$-isométriques s'il existe un isomorphisme de $\mathbb{Z} G$-modules $\phi: R \rightarrow R^{\prime}$ tel que $b(x, y)=$ $b^{\prime}(\phi(x), \phi(y))$. On notera $R \sim R^{\prime}$ et on parlera aussi d'isométrie équivariante. Nous notons par $\bigoplus$ une somme orthogonale.

\subsection{Corps de nombres}

Soit $K / F$ une extension de corps de nombres. Pour un idéal premier $P$ de $K$ on note $v_{P}(J)$ la valuation en $P$ de l'idéal $J$. Aussi $D(K / F)$ est la différente de l'extension $K / F$, définie comme étant l'inverse de l'idéal dual de l'anneau des 
entiers $\mathbb{Z}_{K}$ pour la forme trace

$$
\operatorname{Tr}_{K / F}: K \times K \rightarrow F, \quad \operatorname{Tr}_{K / F}(x, y):=\text { trace }_{K / F}(x y) .
$$

Dans le cas où $K / F$ est une extension galoisienne de groupe de Galois $G$ la forme trace est $G$-équivariante et tout idéal stable sous l'action de $G$ est un $\mathbb{Z}_{F} G$-réseau.

\subsection{Rappels sur l'algèbre $\mathbb{Q} G$ pour $G$ cyclique d'ordre $p^{n}$}

Dans toute la suite $p \neq 2$ est un nombre premier. Soit $G$ un groupe cyclique d'ordre $|G|=p^{n}$. On filtre $G$ par la famille de sous-groupes $H_{i}$ où $H_{i}$ est d'ordre $p^{n-i}$ :

$$
G=H_{0} \supseteq H_{1} \supseteq \ldots \supseteq H_{n}=\{1\} .
$$

Considérons les deux familles d'idempotents $\varepsilon=\left\{\varepsilon_{i}\right\}$ et $e=\left\{e_{i}\right\} \quad(0 \leqslant i \leqslant n)$ définies par

$$
\begin{aligned}
& \left|H_{i}\right| \varepsilon_{i}=\sum_{H_{i}} g \quad(0 \leqslant i \leqslant n), \\
& e_{0}=\varepsilon_{0}, \\
& e_{i}=\varepsilon_{i}-\varepsilon_{i-1} \quad(1 \leqslant i \leqslant n) .
\end{aligned}
$$

On vérifie aisément les propriétés suivantes:

(a) $\varepsilon_{i}^{2}=\varepsilon_{i}$ et si $j<i$ alors $\varepsilon_{j} \varepsilon_{i}=\varepsilon_{j}$;

(b) $e$ est l'unique famille d'indempotents (centraux) orthogonaux de l'algèbre $\mathbb{Q} G$, c'est-à-dire que pour tout $i, e_{i}^{2}=e_{i}$, que si $i \neq j$ alors $e_{i} e_{j}=0$ et que de plus on a une décomposition $1=e_{0}+\ldots+e_{n}$ à laquelle correspond la décomposition en algèbres simples de $\mathbb{Q} G$ :

$$
\mathbb{Q} G=\bigoplus_{i} \mathbb{Q} G e_{i} .
$$

Notons par $\mathbb{Q}\left(p^{i}\right)$ le corps cyclotomique des racines $p^{i}$-èmes de l'unité et par $\mathbb{Z}\left[p^{i}\right]$ son anneau d'entiers. $G$ agit sur $\mathbb{Q}\left(p^{i}\right)$ via multiplication par une racine de l'unité d'ordre $p^{i}$. Ceci donne des isomorphismes de $G$-modules

$$
\chi_{i}: \mathbb{Q} G e_{i} \cong \mathbb{Q}\left(p^{i}\right) \text { et } \mathbb{Z} G e_{i} \cong \mathbb{Z}\left[p^{i}\right]
$$

induits par l'évaluation d'un caractère irréductible $\chi_{i}$ de $\mathbb{Q} G$.

L'ordre maximal de $\mathbb{Q} G$ est

$$
\mathcal{M}=\bigoplus_{i} \mathbb{Z} G e_{i} .
$$

A la décomposition (1.3) correspond la décomposition suivante du groupe des classes localement libres de $\mathcal{M}$ en termes des groupes de classes d'idéaux des corps $\mathbb{Q}\left(p^{i}\right)$ :

$$
\mathrm{Cl}(\mathcal{M}) \cong \bigoplus_{i} \mathrm{Cl}\left(\mathbb{Z}\left[p^{i}\right]\right)
$$

EXEMPLE 1.5. Considérons les formes suivantes.

(a) $V=\mathbb{Q} G$ muni de la forme $b=T_{s}$ définie comme suit: pour $z=\sum_{G} z(g) g$ dans $\mathbb{Q} G$, soit

$$
\bar{z}:=\sum_{G} z(g) g^{-1} \text { et } \quad \operatorname{pr}_{1}(z):=z(1),
$$

alors pour $s=\bar{s}$ on définit $T_{s} \operatorname{par} T_{s}(x, y):=\operatorname{pr}_{1}(s x y)$. 
Remarquons que

$$
|G| \operatorname{pr}_{1}(z)=\operatorname{trace}_{\mathbb{Q} G / Q}(z) .
$$

(b) $V=\mathbb{Q}\left(p^{i}\right)$ muni de la forme

$$
b(x, y)=\operatorname{tr}_{i, 0}(\sigma x, y):=\operatorname{trace}_{\mathbb{Q}\left(p^{i}\right) / \mathbb{Q}}(\sigma x \bar{y}),
$$

où - dénote la conjugaison complexe et $\sigma$ dans $\mathbb{Q}\left(p^{i}\right)$ est tel que $\sigma=\bar{\sigma}$.

Avec ces notations on voit que la décomposition (1.1) est orthogonale pour $T_{s}$ et que-grâce à (1.6)-les isomorphismes (1.2) sont des isométries:

$$
|G| T_{s}(x, y)=\sum \operatorname{tr}_{i, 0}\left(\chi_{i}(s) \chi_{i}(x), \chi_{i}(y)\right) .
$$

\subsection{Les réseaux $A_{K}^{\mu}, A_{K}$ et $M A_{K}$}

Soit $K / \mathbb{Q}$ une extension galoisienne de groupe $G$. Pour la forme trace $\operatorname{Tr}_{K / \mathbb{Q}}$ le dual d'un idéal $J$ de $K$ est l'idéal

$$
J^{*}=J^{-1} D(K / \mathbb{Q})^{-1} .
$$

On voit que, si le degré de l'extension $K / \mathbb{Q}$ est impair, alors il existe un unique idéal $A_{K}=A(K / \mathbb{Q})$ dans $K$ avec $A_{K}^{2}=D(K / \mathbb{Q})^{-1}$ : utiliser par exemple la formule donnant la valuation de la différente $D(K / \mathbb{Q})$ en termes des groupes de ramification

$$
v_{P}(D(K / \mathbb{Q}))=\sum_{-1<j}(|G(j, P)|-1)
$$

(voir [14, IV.1, Proposition 4]). Il est clair par (1.7) que $A_{K}$ est un $\mathbb{Z} G$-réseau unimodulaire.

Comme annoncé, le but de cet article est de décrire la classe de $\mathbb{Z} G$-isométrie du $\mathbb{Z} G$-réseau $\left(A_{K}, \operatorname{Tr}_{K / \mathbb{Q}}\right)$ dans le cas où l'extension satisfait aux conditions $(0.2)$ de l'introduction. On suppose désormais que $K / \mathbb{Q}$ est une telle extension. D'après [6-8], dans cette situation $A_{K}$ n'est même pas $\mathbb{Z} G$-projectif (sauf si $n=1$ ), c'est pourquoi on essaye d'abord de décrire la structure de $A_{K}$ sur l'ordre maximal $M$.

Definition 1.6. On note $M A_{K}$ le plus petit $M$-module contenant $A_{K}$ qui soit contenu dans $K$ et on note $A_{K}^{\mathcal{M}}$ le plus grand $\mathcal{M}$-module contenu dans $A_{K}$ :

$$
A_{K}^{\mathcal{M}} \subseteq A_{K} \subseteq M A_{K} \text {. }
$$

Les $M$-modules $M A_{K}$ et $A_{K}^{\mu}$ sont des $\mathbb{Z} G$-réseaux pour la forme trace; nous allons voir au $\S 3$ qu'ils sont une bonne approximation de $\left(A_{K}, \operatorname{Tr}_{K / Q}\right)$. En effet de manière générale on peut montrer que $\left[A_{K}: A_{K}^{M}\right]$ et $\left[M A_{K}: A_{K}\right]$ sont des puissances de $p$ qui diminuent quand l'indice de ramification de $p$ dans $K$ augmente.

\section{Propriétés des $\mathbb{Z} G$-réseaux $M A_{K}$ et $A_{K}^{\mathcal{M}}$}

Ce paragraphe décrit les réseaux $M A_{K}$ et $A_{K}^{\mathcal{M}}$ définis plus haut. En particulier nous donnons ici leur décomposition en somme de $\mathbb{Z} G$-réseaux indécomposables et nous faisons le calcul de leur discriminant.

Proposition 2.1. (1) Comme $A_{K}$ est unimodulaire on a

$$
\left(A_{K}^{\mathcal{M}}\right)^{*}=M A_{K} \text {. }
$$


(2) A la décomposition (1.3) de l'ordre maximal correspond la décomposition

$$
A_{K}^{\mathcal{M}}=R_{0} \oplus \ldots \oplus R_{n}
$$

où pour $0 \leqslant i \leqslant n, R_{i}:=e_{i}\left(A_{K}^{\mathcal{M}}\right)$ est un $\mathbb{Z} G$-réseau indécomposable. Aussi

$$
M A_{K}=R_{0}^{*} \oplus \ldots \oplus R_{n}^{*}
$$

(3) Les réseaux $R_{i}$ sont aussi décrits par

$$
R_{i}=A_{K} \cap e_{i}(K), \quad R_{i}^{*}=e_{i}\left(A_{K}\right) .
$$

(4) Pour $0 \leqslant i \leqslant n, R_{i}$ est un $\mathbb{Z} G$-réseau entier, de rang sur $\mathbb{Z}$ égal à: $\varphi\left(p^{i}\right)=p^{i-1}(p-1)$. Le polynôme caractéristique de l'endomorphisme de $\mathbb{Q} R_{i}$ donné par un générateur de $G$ est égal au $p^{i}$-ème polynôme cyclotomique $\phi_{p^{i}}(x)$.

(5) Pour $1 \leqslant i \leqslant n, R_{i}$ est pair.

Démonstration. (1) Il suffit de remarquer que $\left(M A_{K}\right)^{*}$ est aussi un $M$-module et que $\left(M A_{K}\right)^{*} \subseteq A_{K}^{*}=A_{K}$; il s'ensuit que $\left(\mathcal{M} A_{K}\right)^{*} \subseteq A_{K}^{\mu}$ d'où $\left(A_{K}^{\mu}\right)^{*} \subseteq M A_{K}$; la propriété de minimalité de $M A_{K}$ permet de conclure.

(2) De (1.3) on tire la décomposition en modules

$$
A_{K}^{\mu}=\bigoplus_{i} e_{i}\left(A_{K}^{\mu}\right)
$$

Il suffit de montrer que les $R_{i}:=e_{i}\left(A_{K}^{M}\right)$ sont deux à deux orthogonaux: ceci suit du fait que pour $j \neq i, \operatorname{Tr}_{K / Q}\left(e_{i}(x), e_{j}(y)\right)=\operatorname{Tr}_{K / Q}\left(e_{i} e_{j}(x), y\right)=0$ car $e_{i} e_{j}=0$.

(3) Il est clair que $R_{i} \subseteq A_{K} \cap e_{i}(K)$ et par (2.2) il suffit de montrer que chaque $A_{K} \cap e_{i}(K)$ est un $\mu$-module: soit $x_{i}$ dans $A_{K} \cap e_{i}(K)$ et soit $m=\sum_{j} a_{j} e_{j}$ dans $\mu$ (avec $a_{j}$ dans $\mathbb{Z} G$ ); alors $m x_{i}=a_{i} x_{i}$, d'où le résultat car $A_{K}$ est $\mathbb{Z} G$-stable. Pour $R_{i}^{*}$ on procède de façon analogue ou bien on utilise le Lemme 2.4 ci-dessous.

(4) Via les isomorphismes (1.2) on peut considérer $R_{i}$ et $R_{i}^{*}$ comme des sous- $\mathbb{Z}\left[p^{i}\right]$-modules sans torsion dans le $\mathbb{Q}\left(p^{i}\right)$-espace vectoriel $e_{i}(K)$. Aussi $e_{i}(K)=\mathbb{Q} R_{i}$ d'où $\operatorname{rang}_{\mathbb{Z}}\left(R_{i}\right)=\operatorname{dim}_{\mathbb{Q}} e_{i}(K)=\varphi\left(p^{i}\right)$. Si $g$ est un générateur de $G$, alors comme $\mathbb{Q} G e_{i} \cong \mathbb{Q}\left(p^{i}\right)$ on a $\phi_{p^{i}}\left(g e_{i}\right)=0$. Mais $0=\phi_{p^{i}}\left(g e_{i}\right)=\phi_{p^{i}}(g) e_{i}$ car $e_{i}$ est un idémpotent, d'où $e_{i}(K)=\operatorname{ker}\left(\phi_{p^{i}}(g)\right)$. Comme le degré de $\phi_{p^{i}}(x)$ est égal à $\varphi\left(p^{i}\right), \phi_{p^{i}}(x)$ est bien le polynôme caractéristique de $g$ sur $\mathbb{Q} R_{i}=e_{i}(K)$.

(5) Le fait que $R_{i}$ est pair pour $i \geqslant 1$ est une propriété générale des réseaux ayant un automorphisme de polynôme caractéristique $\phi_{p^{i}}(x)$ et découle par exemple du Lemme 1-4 de [1].

Nous calculons maintenant le discriminant de $A_{K}^{\mu}$, en calculant les discrimants des $R_{i}$. Nous allons voir qu'ils ne dépendent que de la ramification sauvage dans $K / \mathbb{Q}$.

Pour le calcul nous introduisons des $\mathbb{Z} G$-réseaux auxiliaires $T_{i}$ définis par:

$$
T_{i}=A_{K} \cap \varepsilon_{i}(K) \quad(0 \leqslant i \leqslant n) .
$$

Soit $K_{i}$ le sous-corps de $K$ fixe par $H_{i}$. Donc $\left[K_{i}: \mathbb{Q}\right]=p^{i}, \varepsilon_{i}(K)=K_{i}$ et aussi

$$
T_{i}=A_{K} \cap K_{i} \text {. }
$$

Notons $\operatorname{Tr}_{i, j}$ la forme trace dans l'extension $K_{i} / K_{j}$, ainsi $\operatorname{Tr}_{n, 0}=\operatorname{Tr}_{K / \mathbb{Q}}$.

Attention: sauf mention explicite du contraire, la forme sur $T_{i}$ est toujours $\operatorname{Tr}_{K / \mathbb{Q}}$ et non pas $\operatorname{Tr}_{i, 0}$. 
Proposition 2.3. (1) Pour $0 \leqslant i \leqslant n, T_{i}$ est un $\mathbb{Z} G$-réseau de rang $p^{i}$.

(2) Comme $\varepsilon_{i}=e_{0}+\ldots+e_{i}$ on a les inclusions

$$
\begin{aligned}
& T_{0} \subseteq T_{1} \subseteq \ldots \subseteq T_{n}=A_{K}, \\
& R_{0} \oplus \ldots \oplus R_{i} \subseteq T_{i}, \\
& T_{i-1} \oplus R_{i} \subseteq T_{i} .
\end{aligned}
$$

(3) Le dual de $T_{i}$ pour $\operatorname{Tr}_{K / Q}$ est

$$
\begin{aligned}
& T_{i}^{*}=\varepsilon_{i}\left(A_{K}\right) . \\
\operatorname{disc}\left(T_{i}\right) & = \begin{cases}1 & \text { si } n \equiv i \bmod 2, \\
p & \text { sinon, }\end{cases} \\
\operatorname{disc}\left(R_{0}\right)= & \begin{cases}1 & \text { si } n \equiv 0 \bmod 2, \\
p & \text { sinon, }\end{cases} \\
\operatorname{disc}\left(R_{i}\right)= & p \text { pour } 1 \leqslant i \leqslant n .
\end{aligned}
$$

(5) $T_{n-2}$ est unimodulaire pour $\operatorname{Tr}_{n, 0}$ d'après (4). La multiplication par $p$ donne une isométrie équivariante entre $\left(T_{n-2}, \operatorname{Tr}_{n, 0}\right)$ et $\left(A\left(K_{n-2} / \mathbb{Q}\right), \operatorname{Tr}_{n-2,0}\right)$.

Démonstration. (1) et (2) sont clairs. Pour montrer (3) nous allons appliquer le lemme suivant à $R=A_{K}$ et $f=\varepsilon_{i}$.

Lemme 2.4. Soit $R$ un réseau dans un $\mathbb{Q}$-espace vectoriel $V$ muni d'une forme $b$ et soit $f$ un endomorphisme de $V$. On définit le transposé 'f de $f$ en imposant

$$
b(f(x), y)=b\left(x,{ }^{t} f(y)\right)
$$

pour tout $x$ et y de $V$. Alors $f(R)$ est un réseau dans $\operatorname{Im}(f)$ de dual

$$
(f(R))^{*}=\left({ }^{\prime} f\right)^{-1}\left(R^{*}\right) \cap \operatorname{Im}(f) .
$$

Démonstration du Lemme 2.4. Elle suit des équivalences

$$
\begin{aligned}
& x \in f(R)^{*} \Leftrightarrow x \in \operatorname{Im}(f) \text { et } b(x, f(R)) \subseteq \mathbb{Z}, \\
& b(x, f(R)) \subseteq \mathbb{Z} \Leftrightarrow b\left({ }^{t} f(x), R\right) \subseteq \mathbb{Z} \Leftrightarrow x \in\left({ }^{f} f\right)^{-1}(R)^{*} .
\end{aligned}
$$

La Proposition 2.3(3) suit du lemme car

$$
\left(A_{K}\right)^{*}=A_{K} \quad \text { et } \quad{ }^{t} \varepsilon_{i}=\varepsilon_{i}
$$

d'où $\left(\varepsilon_{i}\left(A_{K}\right)\right)^{*}=\left(\varepsilon_{i}^{-1}\left(A_{K}\right)\right) \cap \varepsilon_{i}(K)=A_{K} \cap \varepsilon_{i}(K)=T_{i}$ car pour $x$ dans $\varepsilon_{i}(K)$, $\varepsilon_{i}(x)=x$.

(4) Par définition:

$$
\begin{aligned}
\operatorname{disc}\left(T_{i}\right)=\left[T_{i}^{*}: T_{i}\right] & =\left[1 / p^{n-i} \operatorname{Tr}_{n, i}\left(A_{K}\right): A_{K} \cap K_{i}\right] \\
& =\left(N_{i, 0}\left(A_{K} \cap K_{i}\right)\right) /\left(N_{i, 0}\left(1 / p^{n-i} \operatorname{Tr}_{n, i}\left(A_{K}\right)\right)\right)
\end{aligned}
$$

où $N_{i, 0}$ est la norme dans $K_{i} / \mathbb{Q}$.

Rappelons le lemme suivant (voir [16, p. 155]).

Lemme 2.6. Soit $J$ un idéal fractionnaire dans une extension galoisienne $K / F$, qui soit stable sous l'action de $\mathrm{Gal}(K / F)$. Alors

(a) $J$ est de la forme $J=\Pi_{p} \psi(p)^{s(p)}$ où $p$ parcourt les premiers de $F$ et $\psi(p)$ est défini par: $p \mathbb{Z}_{K}=(\psi(p))^{e(p)}$ 
(b) si $P$ est un premier divisant $p$ dans $K$ et $\delta=v_{p}(D(K / F))$, alors

et

$$
v_{p}\left(\operatorname{Tr}_{K / F}(J)\right)=[(\delta+s) / e]
$$

$$
v_{p}(J \cap F)=1+[(s-1) / e]
$$

où $[x]$ dénote le plus grand entier plus petit ou égal à $x$.

Remaroue 2.7. Dans ce lemme l'hypothèse que $J$ est stable par $G a l(K / F)$ est essentielle.

Calculons la valuation de la différente dans notre situation (0.2).

Lemme 2.8. Soit $K_{n} / \mathbb{Q}$ une extension sujette aux conditions $(0.2)$ et soit $K_{i}$ le sous-corps de $K_{n}$ tel que $\left[K_{i}: \mathbb{Q}\right]=p^{i}$. Pour $0 \leqslant i \leqslant n$, notons: $P(i)$ l'unique idéal premier de $K_{i}$ au-dessus de $p$, et $\delta_{i}$ (respectivement $\delta_{i, j}$ ) la valuation en $P(i)$ de la différente de $K_{i} / \mathbb{Q}$ (respectivement $\left.K_{i} / K_{j}\right)$. Alors

(1) $\delta_{i}=(i+1) p^{i}-2-\left(p^{i}-p\right) /(p-1)$,

(2) $\delta_{i, j}=\delta_{i}-p^{i-j} \delta_{j}$.

Le lemme suit d'un calcul standard utilisant la formule (1.8) et de la propriété de transitivité de la différente.

Valuation en $q \neq p$ de disc $\left(T_{i}\right)$. Tout premier $q \neq p$ se ramifie modérément dans $K=K_{n} / \mathbb{Q}$, donc la valuation $d$ de la différente $D(K / \mathbb{Q})$ en $\psi(q)$ est $d=e-1$ (voir Lemme 2.6 pour les notations). D'où il suit-par définition de $A_{K}$ que la valuation de $A_{K}$ en $\psi(q)$ est $-\frac{1}{2}(e-1)$. Du Lemme 2.6, on tire alors pour $Q$ au-dessus de $q$ :

$$
v_{\mathcal{Q}}\left(\operatorname{Tr}_{n, i}\left(A_{K}\right)\right)=v_{Q}\left(A_{K} \cap K_{i}\right)
$$

Par conséquent $q \neq p$ ne divise pas $\operatorname{disc}\left(T_{i}\right)$.

Valuation en $p$ de $\operatorname{disc}\left(T_{i}\right)$. D'après (2.5), et comme $p$ se ramifie totalement dans $K / \mathbb{Q}$, on a

$$
v_{p}\left(\operatorname{disc}\left(T_{i}\right)\right)=p^{i}(n-i)+v_{P(i)}\left(A_{K} \cap K_{i}\right)-v_{P(i)}\left(\operatorname{Tr}_{n, i}\left(A_{K}\right)\right) .
$$

Par le Lemme 2.8, $\delta_{n, i}=\delta_{n}-p^{n-i} \delta_{i}$. Par définition $v_{P(n)}\left(A_{K}\right)=-\frac{1}{2} \delta_{n}$ et l'indice de ramification de $P(i)$ dans $K / K_{i}$ est $e_{n, i}=p^{n-i}$. Donc le Lemme 2.6 entraîne que

$$
v_{p}\left(\operatorname{disc}\left(T_{i}\right)\right)=p^{i}(n-i)+\delta_{i}-2\left[\delta_{n} / 2 p^{n-i}\right]
$$

et de l'expression pour $\delta_{n}$ du Lemme 2.8 on obtient alors

$$
\left[\delta_{n} / 2 p^{n-i}\right]= \begin{cases}\frac{1}{2}\left((n+1) p^{i}-\left(p^{i}-p\right) /(p-1)\right)-1 & \text { si } n \equiv i \bmod 2, \\ \frac{1}{2}\left((n+1) p^{i}-\left(p^{i}-1\right) /(p-1)\right)-1 & \text { sinon. }\end{cases}
$$

En remplaçant dans (2.9) on obtient le résultat voulu.

Calcul de $\operatorname{disc}\left(R_{i}\right)$. On a vu à la Proposition 2.3(2) que

$$
T_{i-1} \oplus R_{i} \subseteq T_{i} \subseteq T_{i}^{*} \subseteq T_{i-1}^{*} \oplus R_{i}^{*},
$$

donc $\operatorname{disc}\left(T_{i-1}\right) \operatorname{disc}\left(R_{i}\right)=\left[T_{i}: T_{i-1} \oplus R_{i}\right]^{2} \operatorname{disc}\left(T_{i}\right)$ et il suffit de montrer

$$
\left[T_{i}: T_{i-1} \oplus R_{i}\right]=\operatorname{disc}\left(T_{i-1}\right) \text {, }
$$


car alors $\operatorname{disc}\left(R_{i}\right)=\operatorname{disc}\left(T_{i}\right) \operatorname{disc}\left(T_{i-1}\right)=p$. Pour $i=0$ on a $e_{0}=\varepsilon_{0}$ donc $R_{0}=T_{0}$. Pour $i \geqslant 1$ montrons

$$
T_{i} /\left(T_{i-1} \oplus R_{i}\right) \cong T_{i-1}^{*} / T_{i-1} .
$$

Soit $f$ l'application de $T_{i}$ dans $T_{i-1}^{*} / T_{i-1}$, telle que $f(x)=\varepsilon_{i}(x) \bmod T_{i-1}$. Alors $f$ est surjective car: si $T_{i}$ est unimodulaire alors $\varepsilon_{i-1}\left(T_{i}\right)$ est le dual de $T_{i} \cap \varepsilon_{i-1}(K)=A_{K} \cap K_{i-1}=T_{i-1}$ et si $T_{i} \neq T_{i}^{*}$ alors $T_{i-1}=T_{i-1}^{*}$. Calculons le noyau de $f$ : pour $x$ dans $T_{i}$, comme $x=\varepsilon_{i}(x)=\varepsilon_{i-1}(x)+\varepsilon_{i}(x)$, on a les équivalences

$$
\varepsilon_{i-1}(x) \in T_{i-1} \quad \Leftrightarrow \quad e_{i}(x) \in R_{i} \quad \Leftrightarrow \quad x \in T_{i-1} \oplus R_{i} .
$$

Ainsi (2.10) est démontré.

(5) $T_{n-2}$ est unimodulaire pour $\operatorname{Tr}_{n, 0}$ par le point (4), donc $p T_{n-2}$ est unimodulaire pour $\operatorname{Tr}_{n-2,0}$ car si $x$ est dans $K_{n-2}$ alors $\operatorname{Tr}_{n, 0}(x)=p^{2} \operatorname{Tr}_{n-2,0}(x)$. Comme $p T_{n-2}$ est un idéal, il est égal à $A\left(K_{n-2} / \mathbb{Q}\right)$.

Ceci achève la démonstration de la Proposition 2.3.

Remarque 2.11. Les $R_{i}$ ont un discriminant minimal, dans le sens où un réseau quelconque ayant les propriétés de $R_{i}$ énoncées à la Proposition 2.1(4) a pour discriminant un multiple de $p$ (voir $\S 4$, Remarque 4.5). En particulier pour $i \geqslant 1$ les $R_{i}$ ne pouvaient pas être unimodulaires.

\section{Réduction à la détermination des réseaux $R_{i}$ et $R_{i}^{*}$}

On garde les notations précédentes; en particulier $K / \mathbb{Q}$ satisfait aux conditions (0.2), $K_{i}$ est l'unique sous-corps de $K=K_{n}$ de degré $p^{i}$ sur $\mathbb{Q}$ et $\operatorname{Tr}_{i, j}$ dénote la trace dans $K_{i} / K_{j}$. Les réseaux $R_{i}$ et $R_{i}^{*}$ ont été définis au paragraphe précédent (voir Proposition 2.1).

THÉORÈME 3.1. Si $n \geqslant 2$ on a une $\mathbb{Z} G$-isométrie

$$
\left(A_{K}, \operatorname{Tr}_{K / \mathbb{Q}}\right) \sim\left(A\left(K_{n-2} / \mathbb{Q}\right), \operatorname{Tr}_{n-2,0}\right) \oplus\left(B_{n}, \operatorname{Tr}_{K / \mathbb{Q}}\right)
$$

où $\left(B_{n}, \operatorname{Tr}_{K / Q}\right)$ est un $\mathbb{Z} G$-réseau unimodulaire de rang $p^{n}-p^{n-2}$. On pose $B_{1}=A\left(K_{1} / \mathbb{Q}\right)$. Alors pour tout $n \geqslant 1$,

$$
R_{n-1} \oplus R_{n} \subseteq B_{n}
$$

et la classe d'isométrie équivariante de $B_{n}$ est déterminée par celles de $R_{n-1}$ et $R_{n}$.

A la Proposition 6.6 nous donnerons une décomposition de $A_{K}$ en termes des réseaux $B_{i}$.

Il est clair que, par récurrence sur $n$, le Theorème 0.3 suit du Théorème 3.1 une fois que l'on aura montré-au §5-que la classe d'isométrie des $R_{i}$ et $R_{i}^{*}$ ne dépend que des conditions (0.2).

Démonstration. Nous savons par la Proposition 2.3 que pour tout $n$ le $\mathbb{Z} G$-réseau $\left(T_{n-2}, \operatorname{Tr}_{K / \mathbb{Q}}\right)$ est unimodulaire: rappelons que $T_{n-2}:=A_{K} \cap K_{n-2}$. D'après un lemme connu $[12,1.3 .1]$ l'inclusion $T_{n-2} \subseteq A_{K}$ donne la décomposition en $\mathbb{Z} G$-réseaux

$$
\left(A_{K}, \operatorname{Tr}_{K / \mathbb{Q}}\right) \sim\left(T_{n-2}, \operatorname{Tr}_{K / \mathbb{Q}}\right) \oplus\left(B_{n}, \operatorname{Tr}_{K / \mathbb{Q}}\right)
$$


où $B_{n}$ est le complément orthogonal de $T_{n-2}$ dans $A_{K}$ formé des éléments $x$ de $A_{K}$ tels que $\operatorname{Tr}_{K / Q}\left(x T_{n-2}\right)=0$. La Proposition 2.3(5) donne l'isométrie

$$
\left(T_{n-2}, \operatorname{Tr}_{K / \mathbb{Q}}\right) \sim\left(A\left(K_{n-2} / \mathbb{Q}\right), \operatorname{Tr}_{n-2,0}\right)
$$

et il ne nous reste qu'à montrer que $B_{n}$ a les propriétés voulues.

Remarquons que $B_{n}$ est unimodulaire puisque $A_{K}$ et $T_{n-2}$ le sont. Comme $R_{n-1} \oplus R_{n}$ est inclus dans l'orthogonal de $K_{n-2}$ (pour $\operatorname{Tr}_{K / \mathbb{Q}}$ ) on a bien

$$
R_{n-1} \oplus R_{n} \subseteq B_{n}=B_{n}^{*} \subseteq R_{n-1}^{*} \oplus R_{n}^{*} .
$$

Montrons qu'à $\mathbb{Z} G$-isométrie près il y a un seul réseau $B_{n}$ vérifiant ces inclusions. Pour tout $i \geqslant 1, \operatorname{disc}\left(R_{i}\right)=p$ donc $R_{i}^{*} / R_{i} \cong \mathbb{F}_{p}$. La forme $\operatorname{Tr}_{K / \mathbb{Q}}$ induit sur ce quotient une forme non-nulle que nous noterons

$$
b_{i}: R_{i}^{*} / R_{i} \times R_{i}^{*} / R_{i} \rightarrow(1 / p) \mathbb{Z} / \mathbb{Z} \subseteq \mathbb{Q} / \mathbb{Z} .
$$

Les réseaux $B$ unimodulaires vérifiant les inclusions (3.2) correspondent bijectivement aux droites isotropes du quotient $\left(R_{n-1}^{*} \oplus R_{n}^{*}\right) /\left(R_{n-1} \oplus R_{n}\right) \cong \mathbb{F}_{p} \oplus \mathbb{F}_{p}$ muni de la forme $b=b_{n-1} \oplus b_{n}$.

LeMme 3.3. $\mathbb{F}_{p} \oplus \mathbb{F}_{p}$ muni de $b$ possède exactement deux droites isotropes. Les réseaux $B$ et $B^{\prime}$ correspondants sont $\mathbb{Z} G$-isométriques.

Démonstration du lemme. (Voir aussi [9].) Soit $x_{0} \neq 0$ dans $\mathbb{F}_{p} \oplus\{0\}$ et $y_{0} \neq 0$ dans $\{0\} \oplus \mathbb{F}_{p}$. Comme $b\left(x_{0}\right) \neq 0$, une droite isotrope contient forcément un vecteur de la forme $x_{0}+k y_{0}$ avec $k$ dans $\mathbb{F}_{p} \backslash\{0\}$ et tel que

$$
b_{n-1}\left(x_{0}\right)+k^{2} b_{n}\left(y_{0}\right)=0 .
$$

Ceci donne deux choix opposés pour $k$, d'où deux droites. (On a au moins une solution car $B_{n}$ existe!). La $\mathbb{Z} G$-isométrie de $R_{n-1}^{*} \oplus R_{n}^{*}$ qui envoye $(x, y)$ sur $(x,-y)$ passe au quotient et échange les deux droites isotropes, donc aussi les deux réseaux $B$ et $B^{\prime}$ correspondants.

\section{Classification de formes sur les corps cyclotomiques $\mathbb{Q}\left(p^{i}\right)$}

Notations du paragraphe. Dans ce paragraphe on fixe l'entier $i \geqslant 1$ et on pose

$$
\begin{aligned}
& k=\mathbb{Q}\left(p^{i}\right), \quad k^{+}=\mathbb{Q}\left(p^{i}\right) \cap \mathbb{R}, \\
& \mu: \text { groupe des racines } p^{i} \text {-èmes de l'unité dans } k, \\
& \zeta: \text { un générateur de } \mu, \\
& \text { : conjugaison complexe, } \\
& \mathscr{P}=(1-\zeta) \mathbb{Z}_{k}, \quad \beta=(1-\zeta)(1-\bar{\zeta}), \\
& P=\mathscr{P} \bar{P} \cap k^{+}=\beta \mathbb{Z}_{k^{+}}, \quad m(i)=-\frac{1}{2}\left(p^{i-1}+1\right), \\
& \delta=p^{i} \beta^{m(i)}, \quad \operatorname{tr}=\operatorname{trace}{ }_{k / \mathbb{Q}} .
\end{aligned}
$$

Remarquons que $\delta$ est un générateur totalement positif de la différente de l'extension $k^{+} / \mathbb{Q}[17$, chapitre 2].

Rappelons que nous cherchons maintenant à déterminer la classe de $\mathbb{Z} G$ isométrie de $R_{i}$ (pour tout $i \geqslant 1$ ). Le choix d'une racine de l'unité d'ordre $p^{i}$ permet de voir $R_{i}$ comme un $\mathbb{Z}_{k}$-module (Proposition 2.1(4)). Etant de rang 1 sur 
$\mathbb{Z}_{k}, R_{i}$ est isomorphe à un idéal de $k$ qui-par cet isomorphisme—est muni d'une forme satisfaisant les propriétés suivantes: cette forme est

$$
\begin{aligned}
& \text { entière, } \\
& \text { invariante sous l'action de } \mu \text {, } \\
& \text { définie positive, } \\
& \text { de discriminant } p
\end{aligned}
$$

(voir Propositions 2.1 et 2.3). Nous allons décrire les formes ayant ces propriétés. On appellera $\mathbb{Z}_{k}$-réseau un $\mathbb{Z}_{k}$-module muni d'une forme invariante sous $\mu$.

Proposition 4.2. (1) A une forme bilinéaire symétrique $b: k \times k \rightarrow \mathbb{Q}$ invariante par $\mu$ on associe un unique $\alpha$ dans $k^{+}$tel que $b(x, y)=\operatorname{tr}(\alpha x \bar{y})$. On pose $a=\alpha \delta$.

(2) $b$ est définie positive si et seulement si a est totalement positif, i.e. tous les conjugués de a sont positifs.

(3) On note $(J, a)$ le $\mathbb{Z}_{k}$-réseau obtenu en munissant l'idéal $J$ de $k$ de la forme

$$
b(x, y)=\operatorname{tr}(\alpha x \bar{y}) \text { avec } a=\alpha \delta .
$$

Alors $(J, a)$ est entier de discriminant p si et seulement si aJJ $=\mathbb{Z}_{k}$.

(4) Les réseaux $(J, a)$ et $\left(J^{\prime}, a^{\prime}\right)$ sont $\mathbb{Z}_{k}$-isométriques si et seulement si il existe $\lambda$ dans $k$ avec $J^{\prime}=\lambda J$ et $a=\lambda \bar{\lambda} a^{\prime}$.

Démonstration. (Voir aussi [13] et [9].) (1) Pour tout $y$ dans $k$ l'application qui à $x$ associe $b(x, y)$ est une forme $\mathbb{Q}$-linéaire donc, comme la forme trace tr est non-dégénérée, il existe un unique $h(y)$ tel que pour tout $x$ et $y$ dans $k$,

$$
b(x, y)=\operatorname{tr}(x h(y)) \text {. }
$$

Comme $b$ est invariante par $\mu$ et que les puissances de $\zeta$ forment une $\mathbb{Q}$-base pour $k$ on a pour tout $y^{\prime}$ dans $k$,

$$
b\left(y^{\prime} x, y\right)=b\left(x, \bar{y}^{\prime} y\right)
$$

d'où

$$
\operatorname{tr}\left(y^{\prime} x h(y)\right)=\operatorname{tr}\left(x h\left(\bar{y}^{\prime} y\right)\right)
$$

et donc pour tout $y$ et $y^{\prime}$ dans $k, y^{\prime} h(y)=h\left(\bar{y}^{\prime} y\right)$. Soit $\alpha:=h(1)$; alors $\alpha=\bar{\alpha}$ est bien dans $k^{+}$par symétrie de $b$ et on a le résultat voulu.

(2) De façon générale la signature de la forme $\operatorname{tr}(\alpha x \bar{y})$ est égale à

$$
\operatorname{Card}\{\sigma \in \operatorname{Gal}(k / \mathbb{Q}) \mid \sigma(\alpha)>0\}-\operatorname{Card}\{\sigma \in \operatorname{Gal}(k / \mathbb{Q}) \mid \sigma(\alpha)<0\} .
$$

(3) Calculons le dual de $(J, a)$ :

$$
x \in J^{*} \Leftrightarrow \operatorname{tr}(\alpha x \bar{J}) \subseteq \mathbb{Z} \quad \Leftrightarrow \quad x \in(\alpha \bar{J} D(k / \mathbb{Q}))^{-1} .
$$

Donc

$$
J^{*}=(\alpha \bar{J} D(k / \mathbb{Q}))^{-1}=(a(1-\zeta) \bar{J})^{-1}
$$

par la transitivité de la différente, la définition de $\delta$ et le fait que $D\left(k / k^{+}\right)=$ $(1-\zeta) \mathbb{Z}_{k}$.

Le réseau $(J, a)$ est entier si et seulement si $J \subseteq J^{*}$, ce qui équivaut par (4.3) à $a J \bar{J} \subseteq(1-\zeta)^{-1} \mathbb{Z}_{k}$; mais comme $P=\bar{P}$ et $a \in k^{+}$, la valuation en $P$ de $a J \bar{J}$ est 
paire et

$$
a J \bar{J} \subseteq(1-\zeta)^{-1} \mathbb{Z}_{k} \quad \Leftrightarrow \quad a J \bar{J} \subseteq \mathbb{Z}_{k}
$$

Si $(J, a)$ est entier,

$$
\begin{aligned}
\operatorname{disc}(J) & =N_{k / \mathbb{Q}}(J) N_{k / \mathbb{Q}}(a \bar{J}) N_{k / \mathbb{Q}}(1-\zeta) \\
& =p\left[a^{-1} \bar{J}^{-1}: J\right] .
\end{aligned}
$$

Donc le discriminant de $(J, a)$ est $p$ si et seulement si $a^{-1} \bar{J}^{-1}=J$, c'est-à-dire $a J \bar{J}=\mathbb{Z}_{k}$.

(4) Une $\mathbb{Z}_{k}$-isométrie entre $(J, a)$ et $\left(J^{\prime}, a^{\prime}\right)$ est d'abord un isomorphisme de $\mathbb{Z}_{k}$-modules, donc une homothétie de rapport un $\lambda$ dans $k$. Ceci impose $J^{\prime}=\lambda J$. Ecrivons que cette homothétie est une isométrie: pour tout $x, y$ dans $J$ et avec $a=\alpha \delta$ et $a^{\prime}=\beta \delta$,

$$
\operatorname{tr}(\beta(\lambda x)(\bar{\lambda} \bar{y}))=\operatorname{tr}(\alpha x \bar{y})
$$

soit $a^{\prime} \lambda \bar{\lambda}=a$.

Remarque 4.5. L'égalité (4.4) montre que le discriminant de $(J, a)$ est nécessairement un multiple de $p$.

La proposition précédente permet de décrire l'ensemble $Q(J)$ des classes de $\mathbb{Z}_{k}$-isométrie de $\mathbb{Z}_{k}$-formes sur un idéal $J$ fixé ayant les propriétés (4.1).

Soit $\mathrm{Cl}(k)$ le groupe des classes d'idéaux de $k$ et $\mathrm{Cl}\left(k^{+}\right)^{+}$le groupe des classes d'idéaux au sens restreint de $k^{+}$. Soit $N^{+}$le noyau de la norme $\mathrm{de} \mathrm{Cl}(k)$ dans $\mathrm{Cl}\left(k^{+}\right)^{+}$. Alors $N^{+}$est le sous-groupe de $\mathrm{Cl}(k)$ formé des classes d'idéaux [J] telles que $J \bar{J}$ soit principal et engendré par un élément totalement positif.

Soit $E^{++}$le groupe des unités totalement positives de $k^{+}$et soit $E^{* *}$ le groupe des normes d'unités de $k$, qui est aussi le groupe des carrés d'unités de $k^{+}: E^{* *}$ est inclus dans $E^{++}$et nous posons

$$
\mathscr{E}=E^{++} / E^{* *} \text {. }
$$

Corollaire 4.6. Soit fixé un idéal $J$ de $k$ dont la classe [J] appartient à $N^{+}$. Soit a un élément totalement positif de $k^{+}$tel que

$$
a J \bar{J}=\mathbb{Z}_{k} \text {. }
$$

L'application qui à $\left(J^{\prime}, a^{\prime}\right)$ associe $a^{\prime} a^{-1}$ est une bijection de l'ensemble $Q(J)$ sur le groupe $\mathscr{E}$.

Corollaire 4.7. Le nombre de classes de $\mathbb{Z}_{k}$-isométrie de $\mathbb{Z}_{k}$-réseaux entiers de rang 1 , de discriminant $p$, et définis positifs est

$$
c=\operatorname{Card}\left(N^{+}\right)\left[E^{++}: E^{* *}\right] \text {. }
$$

Remarque 4.8. Jacques Martinet a observé que le nombre $c$ du Corollaire 4.7 est égal au quotient du nombre de classes de $k$ par le nombre de classes de $k^{+}$.

\section{Structure galoisienne et forme trace}

ThÉORÈmE 5.1. Soit $K / \mathbb{Q}$ une extension galoisienne soumise au seules conditions (0.2). Alors pour $i \geqslant 1,\left(R_{i}, \operatorname{Tr}_{K / Q}\right)$ est $\mathbb{Z} G$-isométrique à $\left(\mathbb{Z}\left[p^{i}\right], 1\right)$. 
Pour la démonstration de ce théorème nous allons utiliser deux résultats démontrés dans [8]. Le premier est que pour une extension $K / \mathbb{Q}$ satisfaisant $(0.2), M A_{K}$ est libre en tant que $M$-module. Le deuxième est le calcul des valuations de certaines résolvantes liées à $A_{K}$.

Remarque 5.2. [8] traite de la structure de $M A_{K}$ en toute généralité avec comme seule condition la projectivité de $A_{K}$ en tant que $\mathbb{Z} G$-module. Bien que $A_{K}$ ne soit pas projectif ici, on peut néanmoins appliquer les résultats de [8], car dans la situation qui est la notre, la classe de $\mu A_{K}$ dans $\mathrm{Cl}(\mathcal{M})$ ne dépend que de la ramification modérée: en effet les idéaux au-dessus de $p$ dans les corps cyclotomiques $\mathbb{Q}\left(p^{i}\right)$ sont principaux (regarder (1.4)).

Par ce qui vient d'être dit, il existe $a$ dans $M A_{K}$ tel que $M A_{K}=M a$. En étendant à $K$ on obtient $K=\mathbb{Q} G a$ et cette égalité donne l'isomorphisme

$$
\varphi: K=\mathbb{Q} G a \cong \mathbb{Q} G, \quad \varphi(\lambda a)=\lambda .
$$

Si $K$ est muni de la forme $\operatorname{Tr}_{K / \mathbb{Q}}$, on transforme $\varphi$ en isométrie en mettant sur $\mathbb{Q} G$ la forme

$$
T_{s(a)}: \mathbb{Q} G \times \mathbb{Q} G \rightarrow \mathbb{Q}, \quad T_{s(a)}(x, y)=\operatorname{pr}_{1}(s(a) x \bar{y})
$$

où les notations sont celles de l'Exemple 1.5 et $s(a)$ est l'élément de $\mathbb{Q} G$ défini par

$$
s(a)=\sum_{G} \operatorname{Tr}_{K / \mathbb{Q}}(a g(a)) g^{-1} .
$$

D'après l'Exemple 1.5 et avec les notations du $\S 4$ on obtient la

Proposition 5.3. Soit $\psi=\bigoplus_{i} \chi_{i}$ l'isomorphisme entre $\mathbb{Q} G$ et $\bigoplus_{i} \mathbb{Q}\left(p^{i}\right)$ de (1.2). Alors pour tout $i \geqslant 1$ la restriction de $\psi \circ \varphi$ à $R_{i}^{*}$ est une $\mathbb{Z} G$-isométrie de $\left(R_{i}^{*}, \operatorname{Tr}_{K / \mathbb{Q}}\right) \operatorname{sur}\left(\mathbb{Z}\left[p^{i}\right], \sigma_{i}|G|^{-1} \delta_{i}\right)$ où $\sigma_{i}=\chi_{i}(s(a))$. En dualisant on obtient que $\left(R_{i}, \operatorname{Tr}_{K / Q}\right)$ est isométrique à $\left(\mathbb{Z}\left[p^{i}\right],|G|\left(\sigma_{i} \delta_{i} \beta_{i}\right)^{-1}\right)$.

En effet par la Proposition 2.1(3), $R_{i}^{*}=e_{i}\left(A_{K}\right)=\mathbb{Z} G e_{i}(a)$, donc $\psi \circ \varphi\left(R_{i}^{*}\right)=$ $\psi\left(\mathbb{Z} G e_{i}\right)=\mathbb{Z}\left[p^{i}\right]$. Pour calculer le dual de $\left(\mathbb{Z}\left[p^{i}\right], \sigma_{i}^{-1}|G| \beta_{i}^{-1}\right)$ utiliser (4.3) et le fait que $s(a)=\bar{s}(a)$.

Pour démontrer le Théorème 5.1 il suffit donc de vérifier, d'après le Corollaire 4.6, que l'élément $E_{i}=|G|\left(\sigma_{i} \delta_{i} \beta_{i}\right)^{-1}$ est trivial dans le groupe $\mathscr{E}$, c'est-à-dire que $E_{i}$, qui est une unité de $k_{i}^{+}$, est la norme d'une unité de $k_{i}$.

Nous allons décrire l'élément $\sigma_{i}$ en termes de résolvantes. Soit $\chi$ un caractère de $G$ et

$$
(a \mid \chi)=\sum_{G} \chi\left(g^{-1}\right) g(a)
$$

la résolvante de $a$ par rapport à $\chi$.

Lemme 5.4. Dans $\mathbb{Q}\left(p^{i}\right)$ on a l'égalité

$$
\sigma_{i}=\left(a \mid \chi_{i}\right) \overline{\left(a \mid \chi_{i}\right)}
$$

Remarquons que $\left(a \mid \chi_{i}\right) \overline{\left(a \mid \chi_{i}\right)}$ est un élément de $k_{i}$, et même de $k_{i}^{+}$, alors que $\left(a \mid \chi_{i}\right)$ lui est dans $K k_{i}$. 
Le lemme découle de l'égalité suivante en appliquant $\chi_{i}$ :

$$
\begin{aligned}
\left(\sum_{G} h(a) h^{-1}\right)\left(\sum_{G} g(a) g\right) & =\sum_{G \times G} h(a) g(a) g h^{-1} \\
& =\sum_{G \times G} g(a) g k^{-1}(a) k \\
& =\sum_{G} \operatorname{Tr}_{K / \mathbb{Q}}(a h(a)) h^{-1} .
\end{aligned}
$$

Ensuite nous utilisons les résultats de [8] qui entraînent que

$$
\left(a \mid \chi_{i}\right)\left(\tau\left(\chi_{i}^{2}\right) \tau\left(\chi_{i}\right)^{-1}\right)^{-1}
$$

est dans $k_{i}$ et engendre une puissance de l'idéal $\left(1-\zeta_{i}\right) \mathbb{Z}\left[p^{i}\right]$. Ici $\tau(\chi)$ est la somme de Gauss associée à la partie modérée du caractère $\chi$ et $\chi^{2}$ est le caractère de valeurs $\chi^{2}(g):=\chi\left(g^{2}\right)$. On sait que si on pose

$$
f(\chi)=\tau(\chi) \bar{\tau}(\chi) \quad \text { (le conducteur) }
$$

alors, comme $|G|$ est impair, $\chi^{2}$ est conjugué de $\chi$ et on a $f\left(\chi^{2}\right)=f(\chi)$, donc

$$
\tau\left(\chi_{i}^{2}\right) \tau\left(\chi_{i}\right)^{-1} \bar{\tau}\left(\chi_{i}^{2}\right) \bar{\tau}\left(\chi_{i}\right)^{-1}=1 \text {. }
$$

On en déduit qu'il existe $u_{i}$ dans $E_{k_{i}}$ et $n(i)$ un entier relatif tels que $\left(a \mid \chi_{i}\right)=u_{i}\left(1-\xi_{i}\right)^{n(i)}$ et donc

$$
\sigma_{i}=\left(a \mid \chi_{i}\right) \overline{\left(a \mid \chi_{i}\right)}=u_{i} \bar{u}_{i} \beta_{i}^{n(i)} .
$$

En revenant à $E_{i}$,

$$
E_{i}=\left(u_{i} \bar{u}_{i}\right)^{-1} p^{n-i} \beta_{i}\left(\left(p^{i-1}-1\right) / 2\right)-n(i)
$$

mais $E_{i}$ est une unité donc

$$
\frac{1}{2}(n-i)\left(p^{i}-p^{i-1}\right)+\frac{1}{2}\left(p^{i-1}-1\right)-n(i)=0
$$

et

$$
E_{i}=\left(p \beta_{i}^{t(i)}\right)^{n-i} \text { modulo } N\left(E_{k_{i}}\right)
$$

où $t(i)=-\frac{1}{2}\left(p^{i}-p^{i-1}\right)$. Il reste à vérifier que $B:=p \beta_{i}^{t(i)}$ est dans $N\left(E_{k_{i}}\right)$. Mais $p=N_{k_{i}^{+} / \mathbb{Q}}\left(\beta_{i}\right)$ donc $B=\Pi_{\sigma} \sigma\left(\beta_{i}\right) \beta_{i}^{-1}$, où $\sigma$ parcourt $\mathrm{Gal}\left(k_{i}^{+} / \mathbb{Q}\right)$. Si on note encore $\sigma$ un prolongement de $\sigma$ à $k_{i}$,

$$
\sigma\left(\beta_{i}\right) \beta_{i}^{-1}=\left[\left(1-\sigma\left(\zeta_{i}\right)\right)\left(1-\zeta_{i}\right)^{-1}\right] \overline{\left[\left(1-\sigma\left(\zeta_{i}\right)\right)\left(1-\zeta_{i}\right)^{-1}\right]} .
$$

Donc $E_{i}=1$ modulo $N\left(E_{k_{i}}\right)$ et (5.1) est démontré.

Remaroue 5.5. Le résultat de (5.1) est indépendant du choix initial des caractères $\chi_{i}$, ce qui prouve bien que les réseaux $A_{K}$ de deux extensions vérifiant $(0.2)$ sont $\mathbb{Z} G$-isométriques, pour toute identification de leurs groupes de Galois.

Remaroue 5.6. Avec les méthodes de ce paragraphe et les résultats de [10] on peut retrouver-de façon plus directe mais équivalente-le fait que dans une extension cyclique $K / \mathbb{Q}$ de degré premier $p \neq 2$ le discriminant de l'extension classe le réseau $\left(\mathbb{Z}_{K}, \operatorname{Tr}_{K / \mathbb{Q}}\right)$ à isométrie équivariante près (voir [4, Chapter IV]). Ceci sans hypothèse de ramification. Au lieu des résultats de [8] on utilise le Théorème 7 de [10] qui exprime le produit $(a \mid \chi) \overline{(a \mid \chi)}$ des résolvantes associées à $\mathbb{Z}_{K}$ en termes du conducteur de $\chi$. 


\section{Description explicite de $A_{K}$ : systèmes de racines}

Notations du paragraphe. Pour chaque $i$ on reprend les notations du $\S 4$. Si $j \leqslant i$ on pose

$$
\zeta_{j}=\left(\zeta_{i}\right)^{p^{i-j}}
$$

La forme $\operatorname{tr}_{i, 0}\left(\delta_{i}^{-1} x \bar{y}\right)$ sur $\left(\mathbb{Z}\left[p^{i}\right], 1\right)$ sera notée $b_{i}(x, y)$.

\subsection{Propriétés de $\left(\mathbb{Z}\left[p^{i}\right], 1\right)$ pour $i \geqslant 1$}

Pour tout entier $q \geqslant 1$ il existe un unique réseau entier défini positif $\left(\mathbb{A}_{q}, b\right)$ de rang $q$, de discriminant $q+1$ et ayant une base formée de racines, i.e. de vecteurs $x$ avec $b(x, x)=2$. Soit $\left\{e_{i}\right\}$ la base canonique de $\mathbb{B}^{q+1}$. On peut voir $\mathbb{A}_{q}$ plongé dans l'espace euclidien $\mathbb{R}^{q+1}$ muni de la forme standard $\langle 1, \ldots, 1\rangle ;$ une base de $\mathbb{A}_{q}$ est donnée par les $q$ vecteurs

$$
a_{i}=e_{i+1}-e_{i} \quad(1 \leqslant i \leqslant q) .
$$

On définit le système de racines d'un réseau entier (pair, défini positif) $(R, b)$ comme étant le sous-réseau engendré par ses vecteurs $x$ avec $b(x, x)=2$. On le note $\operatorname{Rac}(R, b)$. Rappelons le théorème de structure suivant.

Proposition 6.2 (Witt) [3, chapitre VI, No. 4.2]. Un réseau entier, défini positif $(R, b)$ qui est engendré par des vecteurs $e_{i}$ avec $b\left(e_{i}, e_{i}\right)=2$ est une somme orthogonale de systèmes de racines

$$
\mathbb{A}_{q}, \mathbb{D}_{q}, \mathbb{E}_{6}, \mathbb{E}_{7}, \mathbb{E}_{8} .
$$

Les indices indiquent les rangs respectifs et les discriminants sont

$$
\begin{array}{lll}
\operatorname{disc}\left(\mathbb{A}_{q}\right)=q+1, & \operatorname{disc}\left(\mathbb{E}_{6}\right)=3, & \operatorname{disc}\left(\mathbb{E}_{8}\right)=1, \\
\operatorname{disc}\left(\mathbb{D}_{q}\right)=4, & \operatorname{disc}\left(\mathbb{E}_{7}\right)=2 .
\end{array}
$$

Dans la suite, seule la famille des $\mathbb{A}_{q}$ interviendra directement (cependant il y a une exception pour $p=3$ ). Nous renvoyons à [5, Chapter 4] pour les définitions des autres systèmes de racines irréductibles ainsi que pour une discussion des propriétés de ces réseaux.

Proposition 6.3. $(i=1)(\mathbb{Z}[p], 1)$ est isométrique à $\mathbb{A}_{p-1}$.

Démonstration. Un calcul facile montre que dans la base $\left\{1, \zeta_{i}, \ldots, \zeta_{i}^{p-2}\right\}$ la matrice de la forme $b_{1}$ est bien celle donnée par la base (6.1) avec $q=p-1$.

Proposition 6.4. Pour $0<j<i,\left(\mathbb{Z}\left[p^{i}\right], 1\right)$ contient la somme orthogonale de $p^{i-j}$ copies de $\left(\mathbb{Z}\left[p^{j}\right], 1\right)$.

Démonstration. On définit une unité $u_{i j}$ de $\mathbb{Z}\left[p^{i}\right]$ par

$$
u_{i j}=\left(1-\zeta_{j}\right)\left(1-\zeta_{i}\right)^{-p^{i-j}} \text {. }
$$

Alors l'application $f_{i j}$ de $\mathbb{Z}\left[p^{j}\right]$ dans $\mathbb{Z}\left[p^{i}\right]$ définie par

$$
f_{i j}(x)=x\left(1-\zeta_{i}\right)^{\left(p^{i-j}-1\right) / 2} u_{i j}^{\left(p^{j-1}+1\right) / 2}
$$

est une isométrie de $\left(\mathbb{Z}\left[p^{j}\right], b_{j}\right)$ sur un sous réseau $Z_{j}$ de $\left(\mathbb{Z}\left[p^{i}\right], b_{i}\right)$. La somme orthogonale des $p^{i-j}$ translatés différents de $Z_{j}$ par l'itération de la multiplication par $\zeta_{i}$ est le réseau cherché. 
Notation. On désigne par $n(R)$ la somme orthogonale de $n$ copies du réseau $R$.

Proposition 6.5. Pour $p \neq 3$ et pour $i \geqslant 1$, ainsi que pour $p=3$ et $i=1$ :

$$
\operatorname{Rac}\left(\mathbb{Z}\left[p^{i}\right], 1\right)=\left(p^{i-1}\right)\left(\mathbb{A}_{p-1}\right) \text {. }
$$

Pour $p=3$ et $i \geqslant 2$ :

$$
\operatorname{Rac}\left(\mathbb{Z}\left[3^{i}\right], 1\right)=\left(3^{i-2}\right)\left(\mathbb{E}_{6}\right) .
$$

Démonstration. Par la Proposition 6.2 on sait que $\operatorname{Rac}=\operatorname{Rac}\left(\mathbb{Z}\left[p^{i}\right], 1\right)$ est une somme orthogonale de réseaux $\mathbb{A}_{q}, \mathbb{D}_{q}, \mathbb{E}_{6}, \mathbb{E}_{7}$ ou $\mathbb{E}_{8}$. Comme $\mathbb{Z}\left[p^{i}\right]$ est indécomposable en tant que $\mathbb{Z}\left[p^{i}\right]$-module il ne peut apparaitre dans cette somme qu'un seul type de réseau. Les Propositions 6.3 et 6.4 prouvent que

$$
\left(p^{i-1}\right)\left(\mathbb{A}_{p-1}\right) \subseteq \operatorname{Rac} \subseteq\left(\mathbb{Z}\left[p^{i}\right], 1\right),
$$

donc Rac est de rang $p^{i}-p^{i-1}$ et de discriminant une puissance de $p$. En vue des propriétés des réseaux de la Proposition 6.2, il suit facilement que pour $p \neq 3$ la seule possibilité est bien $\operatorname{Rac}=\left(p^{i-1}\right)\left(A_{p-1}\right)$. Cette analyse donne par contre deux possibilités si $p=3$ et $i \geqslant 2:\left(3^{i-1}\right)\left(\mathbb{A}_{2}\right)$ et $\left(3^{i-2}\right)\left(\mathbb{E}_{6}\right)$. Mais la Proposition 6.4 appliquée avec $j=2$ montre que

$$
\left(3^{i-2}\right)(\mathbb{Z}[9], 1) \subseteq\left(\mathbb{Z}\left[3^{i}\right], 1\right) .
$$

Or (Z्Z $[9], 1)$ est un réseau pair de rang 6 , discriminant 3 et défini positif; le seul réseau ayant ces propriétés est $\mathbb{E}_{6}$ (voir [5, Table 15-9]). Ceci termine la démonstration.

\subsection{Décomposition de $A_{K}$}

Soit $K / \mathbb{Q}$ une extension soumise aux seules conditions (0.2).

Proposition 6.6. Suivant la parité de $n$, on a les $\mathbb{Z} G$-isométries

ou

$$
A_{K} \sim\langle 1\rangle \oplus B_{2} \oplus B_{4} \oplus \ldots \oplus B_{n} \text {, sin est pair }
$$

$$
A_{K} \sim B_{1} \oplus B_{3} \oplus \ldots \oplus B_{n}, \quad \text { si n est impair. }
$$

Ici, pour $i \geqslant 2, B_{i}=B_{i}(p)$ est l'unique réseau unimodulaire contenant un sous-réseau d'indice $p \mathbb{Z} G$-isométrique à

$$
\left(\mathbb{Z}\left[p^{i-1}\right], 1\right) \oplus\left(\mathbb{Z}\left[p^{i}\right], 1\right)
$$

et pour $i=1$,

$$
B_{1} \sim\langle 1, \ldots, 1\rangle .
$$

Démonstration. Elle découle des Théorèmes 3.1 et 5.1. Pour (6.7) remarquons que nous savons par (3.1) que $R_{0} \oplus R_{1} \subseteq B_{1}$, c'est-à-dire $\langle p\rangle \oplus A_{p-1} \subseteq B_{1}$ (d'après (6.3)), de plus le Lemme 3.3 montre que ceci caracterise $B_{1}$ à isométrie près. L'isométrie (6.7) suit alors du fait que $A_{p-1}$ peut être construit comme le complément orthogonal d'un vecteur de longueur $p$ dans $\mathbb{Z}^{p}$ muni de la forme standard $\langle 1, \ldots, 1\rangle$ (voir [5, Chapter 4.6]).

REMARQue 6.8. L'isométrie (6.7) donne une autre démonstration du fait que, si $K / \mathbb{Q}$ est cyclique de degré $p$, alors $\left(A_{K}, \operatorname{Tr}_{K / \mathbb{Q}}\right)$ est $\mathbb{Z} G$-isométrique à $\left(\mathbb{Z} G, T_{1}\right)$ (voir [7]). 
Proposition 6.9. Pour $i \geqslant 2$ les $B_{i}$ sont des $\mathbb{Z} G$-réseaux $\mathbb{Z}$-indécomposables. Leur système de racines est:

$$
\begin{array}{ll}
\left(p^{i-2}+p^{i-1}\right)\left(\mathbb{A}_{p-1}\right) & \text { pour } p \neq 3, \\
\left(p^{i-3}+p^{i-2}\right)\left(\mathbb{E}_{6}\right) & \text { pour } p=3 \text { et } i \geqslant 3, \\
\mathbb{E}_{8} & \text { pour } p=3 \text { et } i=2 .
\end{array}
$$

Démonstration. Le $\mathbb{Q} G$-module $\mathbb{Q} B_{i}$ est isomorphe à $\mathbb{Q}\left(p^{i-1}\right) \oplus \mathbb{Q}\left(p^{i}\right)$ donc la seule décomposition non-triviale de $B_{i}$ en somme de $\mathbb{Z} G$-réseaux aurait la forme $B_{i}=M \oplus N$ où $M$ (respectivement $N$ ) serait un $\mathbb{Z}\left[p^{i-1}\right]$ (respectivement $\mathbb{Z}\left[p^{i}\right]$ )réseau. On a remarqué au point (4.5) que de tels réseaux ont pour discriminant un multiple de $p$; donc une telle décomposition contredirait le fait que $B_{i}$ est unimodulaire. Donc, si $B_{i}$ est $\mathbb{Z}$-décomposable, la seule possibilité est qu'il soit isotypique, i.e. $B_{i}=\left(p^{u}\right) L$ pour un certain réseau $L$ et $u \leqslant i-2$. Dans cette décomposition les copies de $L$ sont permutées circulairement sous l'action d'un générateur $g$ de $G$. Elles sont toutefois stables par $e_{i}$ car $e_{i}$ est un polynôme en $g^{p^{i-1}}$, donc en $g^{p^{u}}$. On en tire que $R_{i}=e_{i}\left(B_{i}\right)=e_{i}(L)^{p^{u}}$ et $\operatorname{disc}\left(R_{i}\right)=p$ implique $p^{u}=1$. Un raisonnement analogue à celui de la Proposition 6.5 donne le système de racines.

\subsection{Exemples numériques}

Les réseaux pairs unimodulaires de rang inférieur ou égal à 24 sont tous connus par un travail de Niemeier [5, Chapter 16]; ils ont tous pour rang un multiple de 8. En dimension 8 le seul est $\mathbb{E}_{8}$. Donc avec les notations de la Proposition 6.6: $B_{2}(3)=\mathbb{E}_{8}$. En dimension 24 il y a 24 réseaux qui sont caracterisés par leur système de racines:

$B_{2}(5)$ a un système de racines de type (6) $\mathbb{A}_{4}$,

$B_{3}(3)$ a un système de racines de type $(4) \mathbb{E}_{6}$.

Il se peut, qu'en général (pour tout $p$ ) $B_{2}$ puisse être caracterisé comme le seul réseau 'invariant' de l'algèbre de Lie $\mathrm{sl}(2, \mathbb{C})$ qui soit unimodulaire indécomposable et qui a un système de racines non-vide (voir [2]).

\section{Bibliographie}

1. E. BAYER-FLUCKIGER, 'Definite unimodular lattices having an automorphism of given characteristic polynomial', Comment. Math. Helv. 59 (1984) 509-538.

2. A. I. Bondal et al., 'Invariant lattices, the Leech lattice and its even unimodular analogues in the Lie algebras $A_{p-1}$, Math. USSR Sbornik 58 (1987) no. 2, 435-465.

3. N. BouRBaKI, Groupes et algèbres de Lie (Hermann, Paris, 1968), chapitres 4-6.

4. P. E. CONNER and R. PERLIS, A survey of trace forms in algebraic number fields (World Scientific, Singapore, 1984).

5. J. H. Conway and N. J. A. SloANe, Sphere packings, lattices and groups (Springer, New York, 1988).

6. B. EREZ, 'Structure galoisienne et forme trace dans les corps de nombres', Thèse, Universite de Genève, 1987.

7. B. EREZ, 'The Galois structure of the trace form in extensions of odd prime degree', J. Algebra 118 (1988) 438-446.

8. B. EREZ, 'The Galois structure of the square root of the inverse different', en préparation.

9. W. FErT, 'On integral representations of finite groups', Proc. London Math. Soc. (3) 29 (1974) 633-683.

10. A. FRÖHLiCH, Galois module structure of algebraic integers, Ergebnisse der Mathematik (3)1 (Springer, Heidelberg, 1983). 
11. A. FröhulCh, Classgroups and Hermitian modules, Progress in Mathematics 48 (Birkhäuser, Boston, 1984).

12. J. Milnor and D. Husemoller, Symmetric bilinear forms, Ergebnisse der Mathematik 73 (Springer, Heidelberg, 1973).

13. H.-G. QUebbemanN, 'Zur Klassifikation unimodularer Gitter mit Isometrie von Primzahlordnung', J. reine angew. Math. 326 (1981) 158-170.

14. J.-P. SERRE, Corps locaux, 3ème édition (Hermann, Paris, 1968).

15. N. Stolzfus, 'Unraveling the integral knot concordance group', Mem. Amer. Math. Soc. 12, 192 (1977) 1-91.

16. S. UlloM, 'Normal bases in Galois extensions of number fields', Nagoya Math. J. 34 (1969) 153-167.

17. L. C. Washington, Introduction to cyclotomic fields, Graduate Texts in Mathematics 83 (Springer, New York, 1982).

Université de Bordeaux I

Laboratoire de Mathématiques

351, Cours de la Libération

F-33405 Talence cedex

France
Section de Mathématiques

Université de Genève

C.P. 240

Rue du Lièvre 2-4

CH-1211 Genève 24

Switzerland 